\title{
ESTUDO COMPARATIVO DA ÁREA OCUPADA PELOS CORPOS PEDUNCULADOS NO CÉREBRO DE DUAS ESPÉCIES DE ABELHAS (HYMENOPTERA, APOIDEA)
}

\author{
Fábio Camargo Abdalla ${ }^{1}$ \\ Carminda da Cruz-Landim ${ }^{2}$
}

\begin{abstract}
COMPARATIVE STUDY OF THE AREA OCCUPIED BY THE CORPORA PEDUNCULATA IN THE BRAIN OF TWO BEE SPECIES (HYMENOPTERA, APOIDEA). The present study compare the size of the corpora pedunculata (mushroom bodies) of Exomalopsis aureopilosa a quasi-social specie and Apis mellifera a eusocial specie of bees. The aim was to correlate the developmental degree of such structures with the behavior complexity. The results show that the female specimens of both species have the corpora pedunculata with same relative size. However the area occupied by the neurones cellular bodies (glomeruli) is greater in the workers of $A$.mellifera. In other way in E. aureopilosa the total size of the corpora pedunculata is larger in females, but the glomeruli area is relatively larger in the male.

KEY WORDS. Hymenoptera, Apoidea, corpora pedunculata, glomeruli, castes, behavior
\end{abstract}

O cérebro dos insetos é um centro de associação que recebe estímulos provenientes dos órgãos dos sentidos e está em contato com a cadeia nervosa ventral através de uma rede de interneurônios. Do cérebro emanam ordens motoras para os músculos das antenas e para as partes posteriores do corpo, passando através das vias descendentes, pré-motoras, que vão aos gânglios da cadeia nervosa ventral. Além disto, o cérebro é a sede dos padrões do comportamento organizado de longa duração, governando suas modificações pela aprendizagem.

O cérebro dos insetos está dividido em três regiões: proto, deuto e tritocérebro, sendo que na primeira região encontram-se os corpos pedunculados.

DUJARDIM (1850) fez as primeiras descrições a respeito dos corpos pedunculados, como estruturas características, localizadas no protocérebro dos insetos. Cada um é formado por um par de cálices de fibras nervosas ligados por um pedúnculo, simetricamente localizados de cada lado do protocérebro e contendo no interior corpos celulares de neurônios.

A idéia de que a sociabilidade dos insetos está relacionada com o tamanho de algumas estruturas cerebrais foi aventada pela primeira vez em estudos sobre castas de formigas por FOREL (1901).

1) Financiado pelo CNPq.

2) Departamento de Biologia, Instituto de Biociências de Rio Claro, Universidade Estadual Paulista. Caixa Postal 199, 13500 Rio Claro, São Paulo, Brasil.

E-mail: fabdalla@rcb000.uesp.ansp.br 
HUBER (1965) verificou que cauterizando partes ou a totalidade dos corpos pedunculados, o comportamento de estridulação dos ortópteros era alterado tanto na capacidade de emissão do estrídulo como na sua percepção.

Numa visão global dos insetos nota-se que os Protura e os Thysanura, que não possuem os corpos pedunculados e os Ephemeroptera, Megaloptera e Lepidoptera, que os possuem pouco desenvolvidos, apresentam um padrão comportamental limitado em relação as ordens filogeneticamente mais avançadas como os Coleoptera e os Hymenoptera, sendo que esta última ordem apresenta o mais complexo padrão comportamental social e possue tanto o cérebro como os corpos pedunculados mais desenvolvidos.

De modo geral, segundo RATZERDORFER (1952) e RENSCH (1959) os insetos considerados mais evoluídos e vivendo em sociedade têm o cérebro e os corpos pedunculados relativamente maiores. Porém Vowless (1955), afirma que a comparação do tamanho destas estruturas não deve ser correlacionada entre espécies filogeneticamente muito separadas, pois, exemplifica, tanto as abelhas (Apis mellifera) como as formigas (Formica $\mathrm{sp}$ ) apresentam capacidade mental equiparável, porém nota-se uma diferença marcante, no tamanho do cérebro, sendo o de Apis muito maior.

ALTEN (1910) e RATZERDORFER (1952) realizaram alguns estudos em vertebrados e também nos insetos, com a finalidade de correlacionar o desenvolvimento de algumas partes do cérebro com o grau de cerebralização do animal, ou complexidade de seu comportamento ou de sua organização social. Admitindo que as partes do cérebro que não estão envolvidas nas atividades associativas mantêm-se mais ou menos constantes nos grupos de animais relacionados (RATZERDORFER 1952), um estudo comparativo das estruturas cerebrais entre espécies relacionadas, juntamente com o conhecimento de sua posição filogenética ou seu grau de sociabilidade poderiam esclarecer qual ou quais estruturas estariam relacionadas com a complexidade do comportamento ou sociabilidade do inseto.

JAWLOWSKI (1960) argumenta que a condição de maior desenvolvimento cerebral e dos corpos pedunculados nos insetos sociais só aparece quando se estudam insetos de grupos diferentes. No caso de insetos filogeneticamente próximos, segundo este autor, parece ocorrer o contrário. Esta opinião parece estar de acordo com os achados de CRUZ-LANDIM \& ZANIBONI (1986) que estudando algumas estruturas cerebrais em várias espécies de abelhas, verificaram certa tendência para maior tamanho relativo dos lobos ópticos e lobos antenais e tendência para menor tamanho dos corpos pedunculados entre as espécies sociais.

Em A. mellifera e formigas (Acromyrmex multicinodis) PANDAZIS (1930) e JAWLOWSKI (1957), verificaram uma diferença no tamanho do cérebro entre as castas, tendo as operárias cérebros maiores. JAWLOWSKI (1957) atribui este fato às diferenças comportamentais entre as castas, já que as operárias desenvolvem atividades mais complexas que a rainha. No caso de $A$. mellifera, os zangões possuem um aumento evidente dos lobos ópticos, sendo a região central do protocérebro mais reduzida, já que nas sociedades matrifiliais os machos têm participação quase nula na organização social da espécie e necessitam de visão acurada para o acasalamento e sua própria sobrevivência. 
Vários métodos de mensuração do grau de desenvolvimento das estruturas cerebrais foram tentados para que se pudesse ter segurança maior a respeito dos dados obtidos. GoosSEN (1949) apresenta uma revisão geral dos métodos usados até a data, RATZERDORFER (1952) propõe que se pesem as diferentes partes do cérebro.

Tendo em vista as controvérsias presentes na literatura sobre o assunto, realizamos um estudo comparativo da área ocupada pelos corpos pedunculados no cérebro de operárias de Apis mellifera (eussocial) e de fêmeas e machos de Exomalopsis aureopilosa (quasissocial), a fim de verificar como essa situação se apresenta. A comparação entre fềmeas e machos da mesma espécie visa caracterizar as possíveis diferenças de estrutura cerebral entre os sexos.

\section{MATERIAL E MÉTODOS}

Foram estudadas operárias da espécie Apis mellifera (Hymenoptera:Apidae) e abelhas fêmeas e machos da espécie Exomalopsis aureopilosa (Hymenoptera:Anthophoridae).

As abelhas foram dissecadas, seus cérebros retirados, fixados em paraformaldeído a $4 \%$ e incluídos em resina histológica. Foram feitos cortes seriados frontais dos cérebros totais, com 5 micrometros de espessura, posteriormente corados com hematoxilina e eosina.

Os cérebros e os corpos pedunculados foram medidos com o auxílio do sistema IMPACT, acoplado a um microscópio óptico comum.

\section{RESULTADOS}

Quanto a morfologia os corpos pedunculados apresentem-se bastante semelhantes em ambos os sexos da espécie Exomalopsis aureopilosa, sendo os cálices mediais mais abertos e rasos que os laterais (Fig. 1). Em Apis mellifera esse dimorfismo entre os cálices não é muito claro, sendo ambos os cálices profundos e não muito abertos.

A porcentagem de área que os corpos pedunculados ocupam dentro do cérebro é praticamente a mesma tanto nas fêmeas de $A$. mellifera como nas de $E$. aureopilosa, $16 \%$. Os machos de E. aureopilosa possuem uma área mais reduzida em comparação com as fêmeas de ambas as espécies, $12 \%$ (Tab. I). No entanto, a área ocupada pela região dos corpos celulares de neurônios, chamada aqui de glomérulo (Fig. 2), mostra-nos que há uma diferença significativa entre as operárias de $A$. mellifera e as fềmeas de $E$. aureopilosa, notamos ainda a mesma área glomerular entre as fêmeas e os machos da espécie quasissocial (Tab. II), o que torna esta área relativamente maior nos machos.

\section{DISCUSSÃO E CONCLUSÕES}

Os comportamentos sociais de um modo geral e especificamente nas abelhas, exigem o desenvolvimento de uma grande eficiência na comunicação entre os 

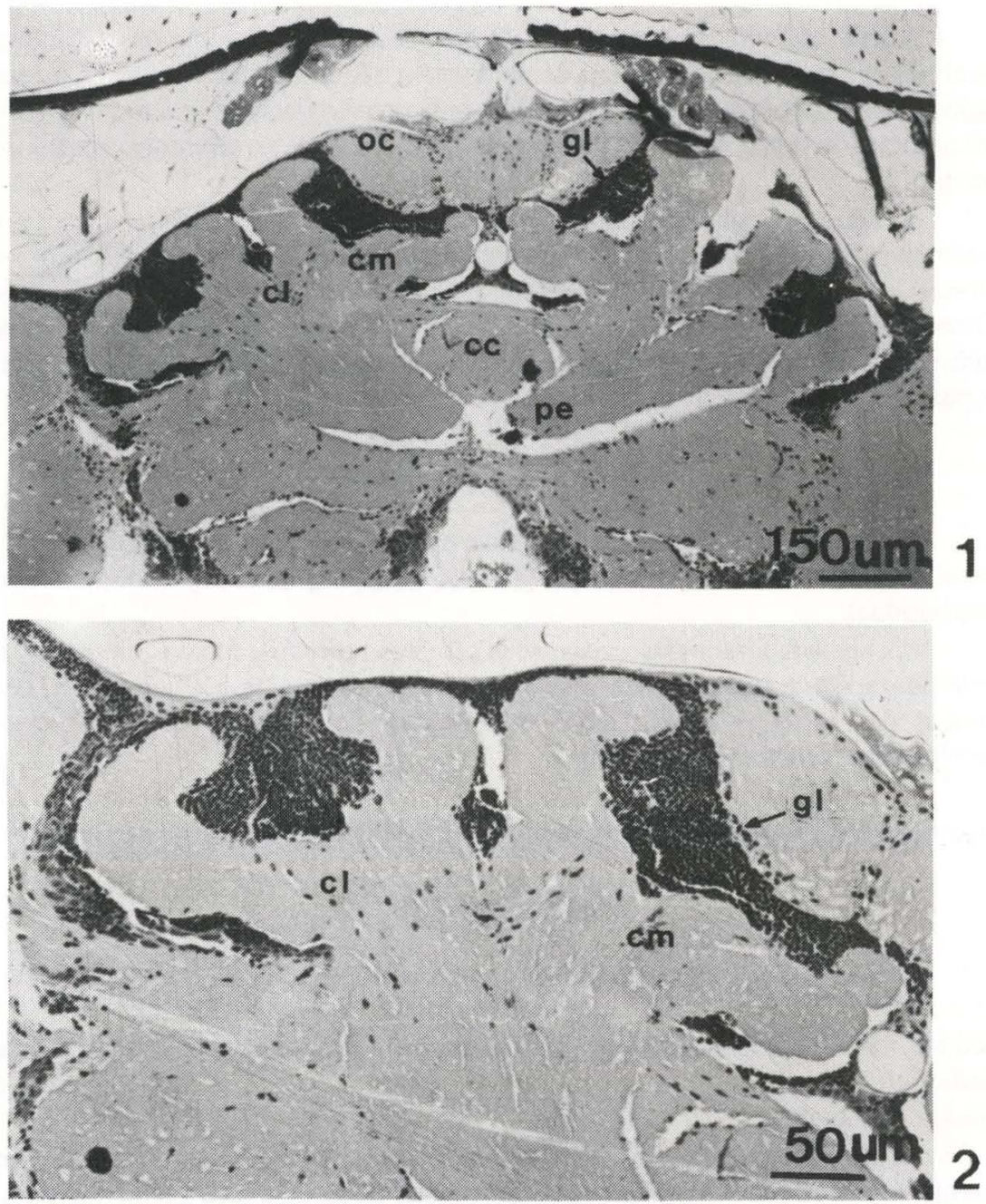

Figs 1-2. (1) Corte frontal mediano dos corpos pedunculados de Exomalopsis aureopilosa, observa-se os ocelos (oc), os corpos pedunculados laterais (cl) e mediais $(\mathbf{c m})$, a área glomerular (gl) e o corpo central (cc); (2) detalhe do corpo pedunculado direito, evidenciando o dimorfismo entre os cálices lateral (cl) e medial $(\mathbf{c m})$, observa-se ainda a região glomerular (gl).

indivíduos. Deste modo, a transmissão, recepção, integração e coordenação dos sinais sociais são de extrema importância na organização social. A divisão de trabalho e a especialização de parte do grupo social em determinadas tarefas se faz imperativa na organização do comportamento social e chega ao extremo de nos himenópteros eussociais originar as castas. Em cada uma destas, por sua vez, ocorre um maior desenvolvimento de habilidades específicas de seus integrantes. Assim, 
em A. mellifera, considerada uma espécie hipersocial (MENZEL \& ERBER 1980), à rainha cabe a reprodução e o "controle" da sociedade, às operárias cabe a defesa, a manutenção do ninho, o cuidado e a alimentação da prole e ao zangão cabe o fornecimento da parte genética complementar para a produção de novas rainhas e operárias. Neste sentido, na rainha houve o desenvolvimento e o "monopólio" dos sinais sociais que irão impôr a integração e o controle da sociedade e o desenvolvimento de uma gama considerável de sinais químicos (os feromônios) torna-se, então, compreensível. São esperadas das operárias não só a capacidade de recepção e resposta aos sinais químicos, mas também o desenvolvimento de habilidades motoras (comportamentais complexas) e integradoras para executar as diferentes tarefas que lhe são impostas na organização social. Porém, para a execução destas tarefas fez-se necessário o maior desenvolvimento cerebral, isto é, da parte coordenadora dos estímulos sensoriais, ou seja, dos corpos pedunculados em particular. Tal tendência é evidenciada nos estudos comparativos mais globais onde se nota que as ordens que apresentam um padrão comportamental menos complexo possuem os corpos pedunculados ausentes ou pouco desenvolvidos em relação as ordens que apresentam um padrão comportamental mais complexo (CHAPMAN 1972). Porém, quando se analisa os corpos pedunculados entre espécies, ou mesmo gêneros, filogeneticamente relacionadas ou muito próximas tal tendência não é consistente, mostrando ocorrer o inverso, ou seja, parece que o tamanho dos corpos pedunculados comparativamente diminuem progressivamente a medida que o grau de complexidade comportamental da espécie aumenta (JAWLOWSKI 1960; CRUZLANDIM et al. 1986).

Tabela I. Área ocupada pelos corpos pedunculados no cérebro de operárias de Apis mellifera e de fêmeas e machos de Exomalopsis aurepilosa.

\begin{tabular}{lcccc}
\hline \multicolumn{1}{c}{ Grupos } & $\begin{array}{c}\text { Porcentagem } \\
\text { média }\end{array}$ & $\begin{array}{c}\text { Coeficiente de } \\
\text { variação }\end{array}$ & Desvio padrão & Teste de Tukey \\
\hline Apis operária & 16,844 & 0,193 & 3,257 & $\mathrm{~A}$ \\
Exomalopis fêmea & 16,518 & 0,090 & 1,650 & $\mathrm{~A}$ \\
Exomalopis macho & 12,340 & 0,212 & 2,595 & $\mathrm{~B}$ \\
\hline
\end{tabular}

*. Letras diferentes indicam diferenças significativas ao nivel de $5 \%$.

Tabela II. Área glomerular dos corpos pedunculados de operárias de Apis mellifera e de fêmeas e machos de Exomalopsis aureopilosa.

\begin{tabular}{lcccc}
\hline \multicolumn{1}{c}{ Grupos } & $\begin{array}{c}\text { Area média } \\
\left(\mu \mathrm{m}^{2}\right)\end{array}$ & $\begin{array}{c}\text { Coeficiente de } \\
\text { variação }\end{array}$ & Desvio padrão & Teste de Tukey \\
\hline Apis operária & 84662 & 0,18 & 15427 & $\mathrm{~A}$ \\
Exomalopis fêmea & 57004 & 0,24 & 13745 & $\mathrm{~B}$ \\
Exomalopis macho & 37422 & 0,10 & 3939 & $\mathrm{~B}$ \\
\hline
\end{tabular}

*. Letras diferentes indicam diferenças significativas ao nível de $5 \%$.

No caso das fêmeas de abelhas que têm colônias com organização dita quasissocial poderíamos esperar que o desenvolvimento dos corpos pedunculados se assemelhasse consideravelmente ao das operárias eussociais, já que em ambas as 
espécies os indivíduos desenvolvem tarefas bastante semelhantes que, portanto, exigiriam um grau de desenvolvimento cerebral equiparável.

A princípio, os resultados da presente pesquisa evidenciavam esta tendência, pois as operárias e as fêmeas das espécies estudadas, mesmo estando filogeneticamente distantes, possuem praticamente a mesma área ocupada pelos corpos pedunculados em seus cérebros. O grau de desenvolvimento dos corpos pedunculados, poderia, portanto, ser atribuido à complexidade do comportamento, como já apontaram alguns dos autores citados.

Concordando com esta interpretação, os machos de E. aureopilosa parecem possuir uma área ocupada pelos corpos pedunculados relativamente menor que a das fêmeas, fato que poderia ser explicado pela menor complexidade de seu comportamento, visto que não há participação direta dos machos na organização social do grupo, como em todas as sociedades matrifiliais. Porém, esta condição pode ser apenas aparente, visto que mesmo tendo a área ocupada pelos corpos pedunculados no cérebro menor que a das fêmeas e operárias de ambas as espécies, têm a área glomerular correspondentemente semelhante a das fêmeas de sua espécie, o que significa um maior tamanho absoluto dos glomérulos ou maior número de neurônios neste sexo. Isto parece indicar uma complexidade de comportamento inesperada, já que os machos, em comparação com as fêmeas, não desempenham tarefas que exijam alta complexidade comportamental (como a construção e a memorização da localização dos ninhos ou o cuidado com a prole), possuindo também uma longevidade bem menor que a das fêmeas, expondo-se menos às pressões ambientais. Esta complexidade provavelmente estaria associada ao acasalamento ou a outros comportamentos de importância vital para os machos, ressaltando o fato de que estes não desfrutam das facilidades que os machos de $A$. mellifera encontram no interior da colônia.

A origem de tal complexidade pode estar na herdabilidade das características filogenéticas do grupo como um todo, assim os machos de posse desta complexidade herdada poderiam desenvolvê-la ou incrementá-la isoladamente. Deste modo, no curso de sua evolução os machos de E. aureopilosa não só estariam submetidos à seleção em grupo, mas também à seleção individual, indicando, por outro lado, intensa participação, mesmo que de modo secundário, na característica do grupo no qual poderiam introduzir características distintas. Neste sentido, é preciso fazer uma revisão mais aprofundada do papel do macho para a estruturação da organização social dos himenópteros, verificando também se o mesmo ocorre com os machos de $A$. mellifera.

Analisando somente a área que engloba os corpos celulares de neurônios (área glomerular), nota-se uma diferença considerável entre as operárias de $A$. mellifera e as fêmeas de E. aureopilosa. Nas operárias de A. mellifera a área glomerular é maior, chegando a ter mais que o dobro da área dos machos e quase $o$ dobro da área glomerular das fêmeas de E. aureopilosa (Tab. II), desta forma, admitindo-se que os corpos celulares em ambas as espécies possuem dimensões semelhantes, podemos afirmar que as operárias de A. mellifera possuem um maior número de neurônios, dado não evidenciado quando analisamos os cálices ou os corpos pedunculados como um todo (cálice e glomérulo). Reduzindo o conceito de 
grau de desenvolvimento cerebral ao número de neurônios da área glomerular, notamos que a espécie hipersocial possue um grau de desenvolvimento maior (Tab. II). Estes dados contradizem, portanto, os resultados apresentados quando se analisa os corpos pedunculados como um todo (Tab. I). Mesmo na espécie E. aureopilosa, há diferenças nas áreas glomerulares entre fêmeas e machos e apesar destas diferenças não serem estatisticamente significativas, o valor biológico, em se tratando de conexões nervosas, deve influir na diferenciação entre estes.

Pode-se concluir, que tanto a espécie hipersocial como a quasissocial, em termos de área ocupada pelos corpos pedunculados, apresentam semelhanças, porém o número de neurônios constituintes da estrutura é maior na primeira, esta verificação indica para $A$. mellifera maior diversidade de vias associativas, o que deve ser fundamental para as complexas interações sociais que devem ocorrer no seu nível de sociabilidade.

$\mathrm{O}$ fato de em alguns casos verificar-se um diminuição no tamanho global dos corpos pedunculados (CRUZ-LANDIM \& ZANIBONI 1986) nas espécies com nível mais alto de sociabilidade em relação as de nível menos complexo, tem que ser reinterpretado à luz desta nova verificação, ou seja, do possível aumento no número de neurônios sem o correspondente aumento global da estrutura. Parece ocorrer que a maior complexidade cerebral não se reflete totalmente em maior tamanho das estruturas que compõem o órgão ou do próprio órgão, o qual poderia ser condicionado por outros fatores, mas com o número de neurônios que o compõem.

\section{REFERÊNCIAS BIBLIOGRÁFICAS}

ALtEN, V.H. 1910. Zur Phylogenie Des Hymenopterengeherns. Jenaische Zschr. F. Naturw. 46: 511-590.

CHAPMAN, R.F. 1972. Nervous System, p.515-542. In: The insects structure and function. London, The English University Press., XXVI+819p.

CRUZ-LANDIM, C. DA \& M.C. ZANIBONI. 1986. Estudo comparativo do tamanho de algumas estruturas cerebrais de abelhas (Hymenoptera: Apoidea). Naturalia, São Paulo, 11/12: 23-32.

DujardiM, F. 1850. Mémoires Sur Le Systime Nerveux Des Insects. Ann. Sc. Nat. 14 (3): 195-205.

FOREL, A. 1901. Die Psychischen Fähigkeiten der Ameisen und einiger anderer

Insekten. Verhandlungen des V. Internationalen Zoologen-Congresses $\mathrm{Zu}$ Berlin, p.141-169.

GoossEN, H. 1949. Untersuchungen an gehirnen verschieden grosser jerveils venwandter coleopteren und hymenopteren. Arten. Zool. Jahrb. Allg. Zool. 62: 1-64.

HUBER, F. 1965. Neural integretion (central nervous system). In: M. ROCKSTEIN (Ed.). The physiology of insect. New York, Academic Press, vol. 2.

JAWLOWSK, H. 1957. Nerve Tracts in bees (Apis mellifera) running from the sight and antenal organs to the brain. Lublim-Polônia, Annalis Universitatis Mariae Curie-Shlodowska, vol. XII, p.307-323. 
1960. On the brain structure of the Symphyta (Hymenoptera). Bull. Acad. Polon. Sci. 7: 255-268.

MENZEL, R. \& J. ERBER. 1980. The Influence of the quantity of reward on the learning performance in honeybees. Behaviour 41: 27-42.

PANDAZIS, G. 1930. Über die relative ausbildung der gehernzentren bei biologish verschie denen ameisenarten. Zeitschrift für Morphologie und Ökologie der Ziere 18 (1-12): 114-169;

RATZERDORFER, C. 1952. Volumetric Indices For The Parts OF The Insect Brain. A Compartive Study In Cerebralization Of Insects. Jour. New. York Ent. Soc. 60: $29-152$.

Rensch, B. 1959. Trends Towards Progress Of Brain And Sense Organs. Cold Spring Harbor Symposia on Quantitative Biology, 24: 291-303.

VOWLES, A. 1955. The structure and connexions of the corpora pedunculata in bees and ants. The Quart. Journ. Micr. Sci. 96: (2):239-255.

Recebido em 07.VI.1996; aceito em 28.XII.1996. 